\title{
MINIMALLY INVASIVE MITRAL VALVE REPAIR SURGERY IN PATIENT WITH SYSTEMIC SCLEROSIS
}

\author{
Tran Thuy Nguyen ${ }^{2,3^{*}}$, Long Hoang Luong ${ }^{1+}$, Nguyen Cong Huu ${ }^{2,3+}$, Do Anh Tien ${ }^{2,3}$, Phan Thao Nguyen ${ }^{2}$, \\ Ngo Thanh Hung ${ }^{2,3}$, Nguyen Hoang Nam ${ }^{2,3}$, Nguyen The Binh ${ }^{2,3}$, Ngoc Thanh Le $^{2,3}$
}

\section{ABSTRACT}

Cardiac involvement is an often fatal and underestimated complication in systemic sclerosis, but only few reports have been published on the surgical intervention of patients with cardiothoracic complication in patient with systemic sclerosis. We hereby present a case of severe mitral valve regurgitation due to infective endocarditis on patient with systemic sclerosis. The patient underwent mitral valve reconstruction and post-operative management of systemic sclerosis.

Keywords: systemic sclerosis, mitral valve regurgitation, infective endocarditis, mitral valve reconstruction.

\section{INTRODUCTION}

Systemic sclerosis (SSc) is a heterogeneous autoimmune disorder that affect multiple organ systems. The disease present with the pathogenic triad of vascular lesion, immune dysregulation and widespread fibrosis of multiple organs ${ }^{1}$. Cardiological involvement are frequently reported in SSc patient ranging from 20-30\% up to $80 \%$ in a series of autopsy ${ }^{23}$. Mitral valve prolapse and regurgitation is often reported with incidence up to $60 \%$ though mostly asymptomatic and without severe hemodynamic consequences 45. Only a few articles reported surgical intervention on SSc patient with cardiological involvement ${ }^{67}$. Thus, we hereby present a case of severe mitral valve regurgitation and infective endocarditis complication on patient with systemic sclerosis, with patient underwent mitral valve reconstruction and post-operative management of systemic sclerosis. The diagnosis, management and surgical consideration are further discussed in depth.

\section{CASE REPORT}

A 64-year-old male patient was admitted with severe mitral valve regurgitation and infective endocarditis. At admission, the patient presented with severe dyspnea; pulmonary hypertension; diffuse skin thickening especially fingers of both hands extending proximally (Figure 1); chest CT scan revealed large effusions (Figure 2). Systemic autoimmune disease was suspected however immediate resolve of cardiological complication was required before further therapy for autoimmune disease.

After thorough discussion and consideration, heart bypass surgery was carried out under general anesthesia. First, we open into

1 Department of Allergy-Immunology and Dermatology, E hospital

2 Department of Cardiovascular and Thoracic Surgery, Cardiovascular Center E Hospital

3 University of Medicine and Pharmacy, Vietnam National university Hanoi

+ These authors contributed equally to this work

* Corresponding author: Nguyen Tran Thuy

Email: drtranthuyvd@gmail.com

Address: E Hospital, 87-89 Tran Cung, Hanoi, Vietnam, 10000

Vietnam National University, 114 Xuan Thuy, Hanoi, Vietnam, 10000

Received: 23 June $2021 \quad$ Accepted: 02 July 2021 
the left atrium through the septum. Upon close inspection of the mitral valve, both leaflets were thickened, large vegetation on anterior segment (A1) causing severed anterior segment chordae tendineae, mitral valve prolapse and regurgitation. Complete resection of the vegetation, vancomycin administration followed by artificial chordal reconstruction and mitral valve repair using mitral annuloplasty ring. The surgical procedures were uneventful, with microbiological testing return result of Staphylococcus aureus, the patient was continued on antibiotic therapy with vancomycin and gentamycin. At one-week post operation, with his heart condition stabilized, the patient was transfer to the department of Allergy-Immunology and Dermatology for diagnosis and management of systemic autoimmune disease.

The diagnosis was made based on the ACREULAR Criteria for Systemic Sclerosis. The patient presented with diffuse distribution of skin sclerosis, clear skin thickening of the fingers on both hands extending proximally, together with other criteria for diagnosis of Systemic Sclerosis, Raynaud's phenomenal; puffy fingers with finger tips ulcers and necrosis, abnormal autoantibody profile. After antibiotic therapy, we confirmed that there was no residual infection, therapy for SSc was initiated with hydroxychloroquine, corticosteroids and cyclophosphamide. The patient was discharged later in a stable condition.

\section{DISCUSSION}

Patient with SSc when presented with cardiac complication is often associated with unfavorable outcome due to the existing vascular lesion, immunological dysregulation and widespread fibrosis ${ }^{2}$. Cardiological complication can manifest as the direct consequent of SSc or secondary due to pulmonary hypertension or nephrotic syndrome. Thus, it is often difficult to distinguish primary from secondary cardiological manifestation in SSc. Due to the combined vascular lesion, immune disorder and potential myocardial fibrosis in SSc, cardiac complication of mitral valve regurgitation in combination with infective endocarditis in this patient translate to a very poor prognosis which require timely intervention and aggressive therapy to resolve endocarditis and the underlying systemic sclerosis.

SSc also involve other immunological and systemic disorders including antiphospholipid syndrome which cause liver cirrhosis, pulmonary hyper tension, arterial and venous thromboses, all of which can further complicate the patient condition and increase morbidity and mortality ${ }^{8}$.

Most patients with SSc, as with other autoimmune disorders underwent immunosuppressive therapy to control symptoms and slow down disease progression, which also increases risk of infection and tissue weakness which further complicate any surgical procedure ${ }^{9}$. Complication of intubation during surgical procedure include bleeding due to telangiectasias and regurgitation due to esophageal dysmotility ${ }^{3}$. The range of motion of the mouth or neck is also reduced making it harder for the tracheal intubation. Due to the difficulty of tracheal intubation, some authors have described using thoracic epidural anesthesia in awake patient with SSc undergoing mitral valve replacement ${ }^{10}$. Surgical intervention needs to follow a minimally invasive approach to reduce risk of complication in Systemic sclerosis, 
where diffuse collagen deposition and myocardial fibrosis can obstruct tissue healing.

This case study, to our knowledge, is the first report of surgical intervention of mitral valve regurgitation due to infective endocarditis in patient which was later diagnosed with systemic sclerosis. The case report highlights the importance of careful consideration and timing of the surgical intervention, antibiotic therapy and subsequent immune suppression therapy for systemic autoimmune disorder. The patient requires longterm follow up to monitor SSc disease progression and any further cardiological complication.

\section{DECLARATION}

\section{Declaration of conflicting interests}

The author(s) declared no potential conflicts of interest with respect to the research, authorship, and/or publication of this article.

\section{Informed consent}

We have obtained the patient's consent to publish his images and clinical information for this study. No identifiable information was included in any form.

\section{Funding}

The author(s) received no financial support for the research, authorship, and/or publication of this article.

\section{REFERENCES}

1. Allanore Y, Simms R, Distler O, et al. Systemic sclerosis. Nat Rev Dis Primers 2015; 1: 15002.

2. Follansbee WP. The cardiovascular manifestations of systemic sclerosis (scleroderma). Current Problems in Cardiology 1986; 11: 244-298.
3. Follansbee W, Miller T, Curtiss E, et al. A controlled clinicopathologic study of myocardial fibrosis in systemic sclerosis (scleroderma). The Journal of rheumatology.

4. Marasini B, Massarotti M, Cossutta R. Scleroderma Heart Disease. Int $J$ Immunopathol Pharmacol 2005; 18: 609-614.

5. Comens SM, Alpert MA, Sharp GC, et al. Frequency of mitral valve prolapse in systemic lupus erythematosus, progressive systemic sclerosis and mixed connective tissue disease. The American Journal of Cardiology 1989; 63: 369-370.

6. Ungprasert P, Wannarong T, Panichsillapakit $\mathrm{T}$, et al. Cardiac involvement in mixed connective tissue disease: A systematic review. International Journal of Cardiology 2014; 171: 326-330.

7. Sponga S, Basso C, Ruffatti A, et al. Systemic sclerosis and aortic valve stenosis: therapeutic implications in two cases of aortic valve replacement: Journal of Cardiovascular Medicine 2009; 10: 560-562.

8. Zuo Y, Shi H, Li C, et al. Antiphospholipid syndrome: a clinical perspective. Chinese Medical Journal 2020; 133: 929.

9. Goodman SM, Figgie MP, MacKenzie CR. Perioperative Management of Patients with Connective Tissue Disease. HSS Jrnl 2011; 7: 72-79.

10. Picozzi P, Lappa A, Menichetti A. Mitral valve replacement under thoracic epidural anesthesia in an awake patient suffering from systemic sclerosis. Acta Anaesthesiol Scand 2007; 51: 644-644. 


\section{FIGURES}

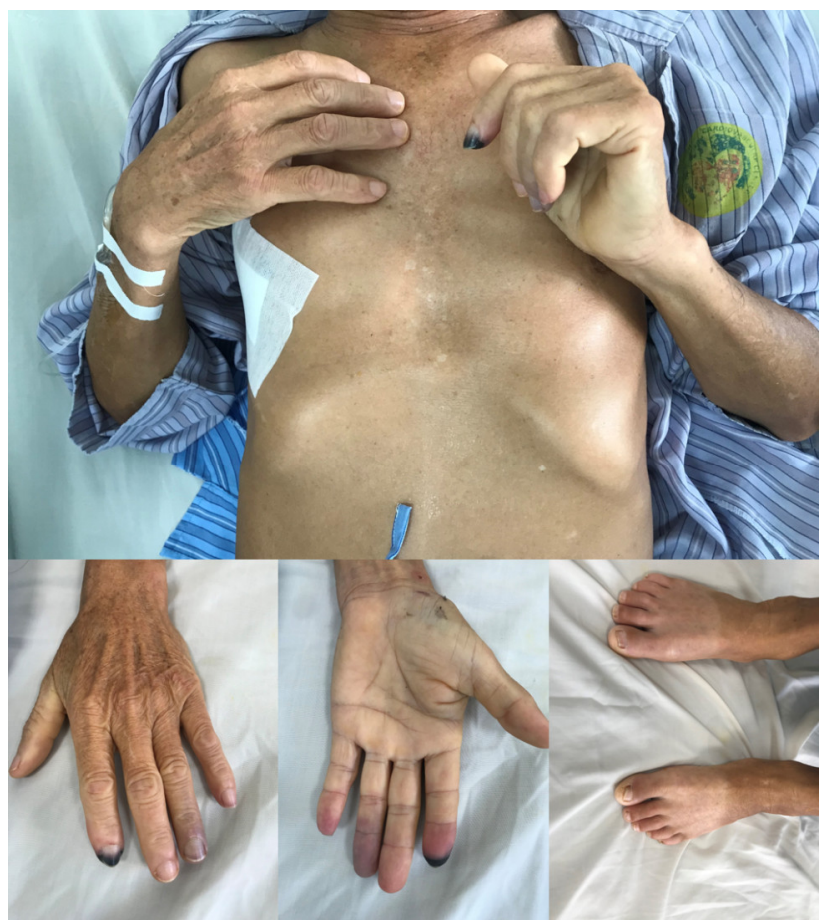

Figure 1: Patient's image post operation; Patient left hand present with Raynaud's phenomenal and II, IV-digit necrosis.

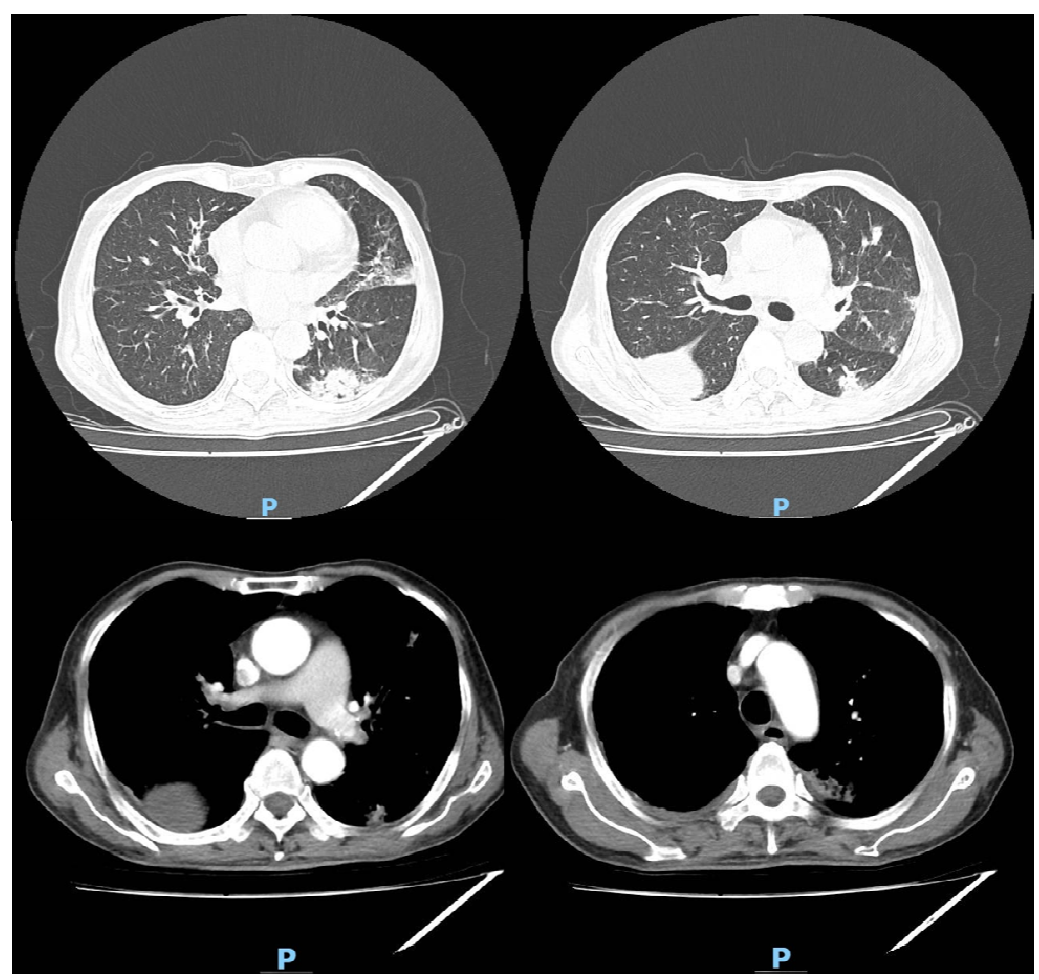

Figure 2: Chest Computed Tomography (CT) 